\title{
Biomedical Signal Transceivers
}

\author{
Reza Fazel-Rezai, Noah Root, Ahmed Rabbi, \\ DuckHee Lee and Waqas Ahmad \\ University Of North Dakota \\ USA
}

\section{Introduction}

With the growing costs of healthcare, the need for mobile health monitoring devices is critical. A wireless transceiver provides a cost effective way to transmit biomedical signals to the various personal electronic devices, such as computers, cellular devices, and other mobile devices. Different kinds of biomedical signals can be processed and transmitted by these devices, including electroencephalograph (EEG), electrocardiograph (ECG), and electromyography (EMG). By utilizing wireless transmission, the user gains freedom to connect with fewer constraints to their personal devices to view and monitor their health condition.

In this chapter, in the first few sections, we will introduce the reader with the basic design of the biomedical transceivers and some of the design issues. In the subsequent sections, we will be presenting design challenges for wireless transceivers, specially using a common wireless protocol Bluetooth. Furthermore, we will share our experience of implementing a biomedical transceiver for ECG signals and processing them. We conclude the discussion with current trends and future work.

The information that is being presented is meant to be applied for all types of biomedical signals. However, some examples are reserved to one type of biomedical signal for simplicity. In this case, the example of an ECG signal and device is used. Even though some sections of the chapter rely heavy on this example, the concepts explored in this chapter can still be extrapolated for other biomedical signals.

\subsection{Types of biomedical signal transceivers}

Different biomedical signal transceiver device types can be designed. There are several distinctions between the types of the devices and their operation. The distinctions can be based upon how the device is powered and how the device communicates. Despite these design differences, the hardware makeup of a biomedical signal transceiver is very standard.

Before going deeper into the details on the types of biomedical signal transceivers, it is important to understand how the device will operate. Typically, a biomedical signal transceiver device will have two main components, the transmitter and the receiver. The transmitter has several sub systems, including: signal acquisition, amplification, filtering, and as the name dictates, transmitter. The receiver subsystem will receive the signal from the transmitter, perform any required analysis on the signal, and then display the results. 
The transmitter is separate from the receiver, such that the transmitter can acquire the bioelectric signal and transmit to another device for remote viewing and analysis.

Existing biomedical transceivers are can be separated into two groups describing how they are powered; Radio Frequency (RF) and battery powered. In RF powered transceivers, an inductive link with external controller allows the transmission of power and commands [2]. A common application of the RF powered transceiver is the transcutaneous neural recording arrays. In battery-powered transceivers, an onboard battery is utilized power source [3]. This battery can be either disposable or rechargeable, depending on the device application. The use of a battery allows using higher frequencies for transmission and improved data rates can be achieved.

Another way to group biomedical transceivers is by their communication style. Biomedical transceivers can communicate either wirelessly or in the traditional wired connection. Not only can the device transmit the biomedical signal, but some devices have communication between the transmitter and receiver for not only biomedical information, but also any feedback or control signals. In this case, both subsystems are acting like transceivers.

\subsection{Applications of biomedical signal transceivers}

Biomedical signal transceivers can be very useful in the monitoring devices and biotelemetry. There are several applications for these devices and their design is as unique as the application. These applications also utilize wireless communications to improve the system and the ease of use.

A health monitoring system which acquires and transmits the vital signals of a patient remotely to a hospital or medical professional can be very useful. This application of biotelemetry can allow for a patient to leave the hospital or clinic, but still have their health monitored remotely. Various bioelectric signals can be recorded from the patient's body and transmitted such as EEG, ECG, body temperature and blood pressure. Biomedical signal transceivers do not have to be limited to just an overall health monitoring device. These transceivers can also have more specific functions that can allow for more in depth analysis, depending on the application.

An ECG monitoring system is a great example of an application of biomedical signal transceiver. When the device is developed wirelessly, patients can monitor their heart signal via a mobile device, while having the electrodes and transmitter attached to their body. Furthermore, a warning system can be designed that can inform the patient about any abrupt abnormality in the heart. As with the health monitoring system, these heart anomalies can also be reported remotely to medical professions who can more appropriately analyze the patient's condition in real time. Another application of biomedical signal transceivers is to monitor the drug and medication usages in the patients remotely.

\section{Analog hardware design}

One of the most important parts of any biomedical signal transceiver is the analog hardware. Using this circuitry the biomedical signal is acquired, filtered, and amplified to an appropriate level. Along with this circuitry, the power for the system needs to be addressed. Finally, safety for both the system circuitry and the patient must be understood and taken care of during design to protect the device as well as the users. 


\subsection{Electrodes}

For all biomedical devices that operate outside of the human body (i.e. non-implantable devices), electrodes are critical components. It is through these electrodes that the bioelectric potentials of the body are collected and transmitted to the measurement and analysis device. In this sense, electrodes are the initial part of any biomedical device.

\subsubsection{Electrode placement}

The placement of the electrodes is dependent on the desired physiological signal. For example, to acquire an ECG signal, the electrodes may be placed in a triangle formation around the heart, creating the Einthoven's triangle. Each of the various bioelectric signals has a standard electrode placement on the body that must be understood and followed before acquiring signals. It is important to have the lead placement correct as noise and distortions will result from electrode misplacement. In some cases, only a small subset of the electrodes is required for signal acquisition. For example, in EEG recording the number of electrode may be from 1 to 128 electrodes. This is dependent on the application or use of the biometric signals by the device.

\subsubsection{Electrode make-up and selection}

An electrode is simply a mechanism that is used to make an electrical connection to a nonmetallic surface. The electrodes have a common makeup, no matter what application or types of signals that are being acquired. Disposable electrodes have a very generic composition and purpose. Using an adhesive, the electrode is attached to the skin, which reduces the risk of noise artifact being introduced into by signal by electrode movement. Additionally, the electrode contains a gel that lowers the skins resistance and is therefore produces a better signal measurement. This allows for the metallic surface to conduct the signal onto the biomedical device.

There are several commercially available electrodes on the market today. The electrode performance will vary from company to company, and from part to part. It is essential to find an electrode that is appropriate for the application, all while keeping quality and price per electrode in mind.

The next step is to develop circuitry to prepare the analog signal for analysis. This will be accomplished by both amplifying and filtering the weak bioelectric signals. These steps are critical for all types of biometric signals.

\subsection{Amplifier and filter design}

When the bioelectric signals are acquired from the human body by the electrodes, the signals are very weak (small amplitudes). Because of their small amplitudes, these signals have little use to any biomedical sensor or system. However, if these signals are amplified to an appropriate level, they can be detected and read accordingly for analysis. The amount of amplification, termed gain, is determined by system specification and is dependent on the signal being measured, and other circuitry requirements. Another critical aspect of the signals that are acquired from the electrodes is the amount of noise in the signal. For proper signal analysis, these errors and noise need to be removed from the signal. The next sections go over the design of amplifiers and filters, all of which accumulates into the filter and amplifier circuitry design. 


\subsubsection{Amplification}

To perform any sort of analysis on a bioelectric signal, the signal needs to be amplified to a level which an analog to digital converter (ADC) can sample the data with a high resolution. As well, the amplifier circuitry needs to include level shifting circuitry such that the signal is positive and has a similar dynamic range as the Analog to Digital conversion. The overall gain is defined by designer based upon the signal requirements.

The first stage in any amplification circuit is the instrumentation amplifier. This amplifier is a critical component for several different reasons, and has many applications outside of just biomedical devices. For one, the instrumentation amplifier acts as a buffer circuit by having large input impedance. This allows for very little current to be drawn from the source. By design, the instrumentation amplifier has a very large Common Mode Rejection Ratio (CMRR). The CMRR simply measures the tendency of the amplifier to reject a signal that is common between the two input pins. This is important for application in biomedical devices since the signal measurement is not coming from one electrode, but actually the difference between two electrodes (i.e. one lead). As well, the instrumentation amplifier will cancel out common noise between the two signals.

There are two ways to implement an instrumentation amplifier; a single IC package or a connection of several separate operational amplifiers (op-amps). Both are viable options, but the choice depends on the cost and efficiency of the choices. In most cases, it can be more efficient to use a single IC package instrumentation amplifier instead of the multiple op amp design. Figure 1 shows an example of cascaded op-amp design for the instrumentation amplifier.

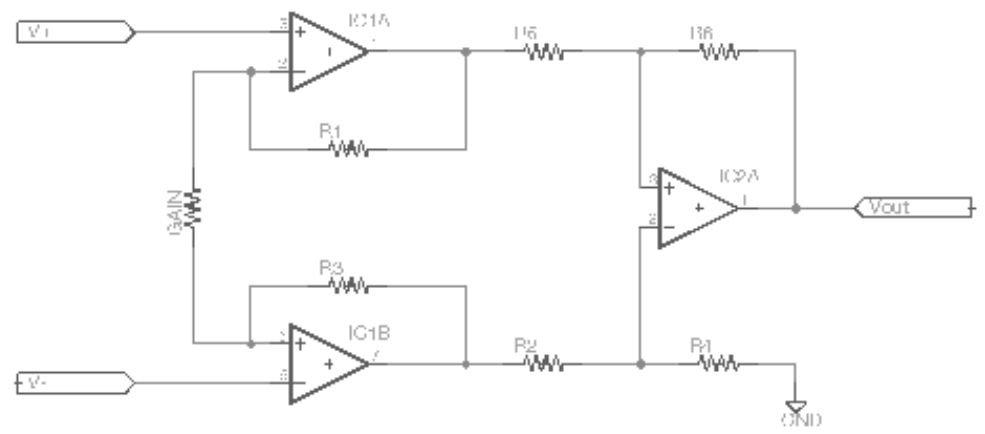

Fig. 1. Instrumentation amplifier layout, utilizing three op-amps

The next stages of the amplifier circuitry are very simple in nature. The amplifier stages only purpose is to increase the amplitude of the bioelectric signals via amplification. Typically, one will employ only non-inverting amplifier configuration so that the signal does not be become inverted or out of phase. The proper number of amplifier stages typically is user defined, but can also be determined based upon the filter configuration. Both of these topics will be covered in the following sections.

The final stage of the amplifier circuitry is a level shifting circuit. The purpose of the level shifting circuit is to shift the negative components of the signal to a positive level. This also shifts up the positive voltage components of the signal as well. This circuit is critical as an analog to digital converter (ADC) on a microcontroller cannot read negative voltages. Thus, 
the signal would not be accurately converted, and the bioelectric information would be lost. There are several ways to implement this circuit; for example, one can use a non-inverting summing amplifier, which is illustrated in Figure 2.

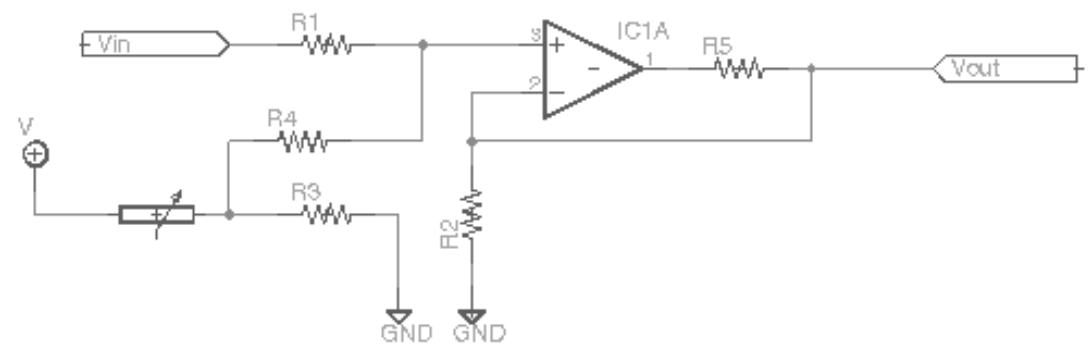

Fig. 2. Summing amplifier design

There are several other designs available, and they all require the use of an amplifier. The level shifting circuit in Figure 3 is a great example of this.

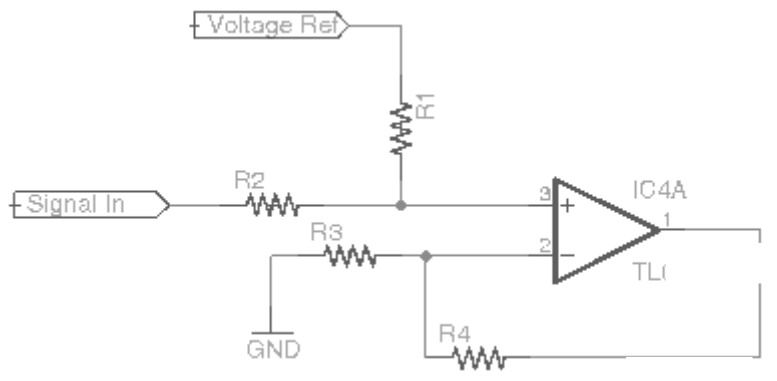

Fig. 3. Level Shifting Circuit

Both of these circuits will allow for the signal to be shifted to an adequate level. To do so, the resistor values will need to be designed such that the values that will allow for proper shifting. These values will also result in gain, if required. For example, if one does not want any gain from the level shifting circuit in Figure 3 (i.e. a gain of 1), simply follow the following guidelines:

$$
\begin{aligned}
& R 1=R 4 \\
& R 2=R 3
\end{aligned}
$$

If other values of gain (A) are required, the following equation should be considered:

$$
\begin{gathered}
\mathrm{A}=(\mathrm{R} 1 / \mathrm{R} 3) \times(\mathrm{R} 3+\mathrm{R} 4) /(\mathrm{R} 1+\mathrm{R} 2) \\
\mathrm{R} 1=\mathrm{R} 3 \\
\mathrm{R} 2=\mathrm{R} 4 \\
\mathrm{~A}=(\mathrm{R} 4 / \mathrm{R} 1)
\end{gathered}
$$


This will allow to tune the circuit as required to shift the voltage. This level shifting circuit should be used if the exact value of shift is known; otherwise, the summing amplifier circuitry should be considered to allow for variance in the shift voltage. The potentiometer in summing amplifier allows the user to vary the voltage divider, which thus varies the shifting voltage level.

\subsubsection{Amplifier selection}

There are many op-amps on the market for use in the amplification of bioelectric signals. However, many of the more common amplifiers, such as the common $741 \mathrm{op}$ amp, do not produce ideal response, especially for bioelectric signals. This is due to the 741 design, or any other op amp that utilizes Bipolar Junction Transistors (BJT) in the first stages of the amplifier. Unlike MOSFET (or other FETs) the BJT will draw current from the signal, thus affecting the signal. As well, there are leakage currents from the BJT that will also hinder the signal. Thus, it can be advantageous to utilize an op amp that uses BiMOS technology. BiMOS is circuit design that use both BJTs and MOSFETs. Similar, BiCMOS can also be used, which simply is BJTs and CMOS. To determine the proper op amp to use in biomedical device one needs to look into the various specifications for the given op amp.

\subsubsection{Filters}

Filters are the other critical component of the analog hardware design for a biomedical device. By removing noise and artifacts from the signal, a precise and more accurate signal can be utilized by the signal analysis code. However, there are a lot of options and configurations for filters, and it can be tricky to determine what is necessary for the application at hand.

Before designing a filter, one needs to determine the frequency range of the bioelectric signals that are being measured. This is critical so that one can determine the required frequency response of the analog filters. Once this is determined, then the filters can be designed.

One of the most important filters, no matter what the frequency range of the bioelectric signal is, is the $60 \mathrm{~Hz}$ (or $50 \mathrm{~Hz}$ outside of North America) notch filter, also known as a band stop filter. This filter removes the noise that is produced from the common AC wall outlet. There are several ways to design a notch filter, with both passive and active designs. The effectiveness of the filter depends on the design. The passive filter designs will not be as exact, and the cut off frequency will vary over time (passive components will vary over time). This can and will affect the signal integrity over time. If there is a substantial enough drift, actually information will be attenuated with the $60 \mathrm{~Hz}$ being freely passed. Active filters, even with their power requirements, are by far the best option for most biomedical device application. One very effective and efficient design is to utilize Texas Instruments' Universal Active Filter, the UAF42, in a notch filter configuration. This design is laid out in the data sheet for the component, which explains the proper design for a $60 \mathrm{~Hz}$ notch filter with the chip and selected resistor values. There are several other active filter design and options that can be utilized to attenuate the $60 \mathrm{~Hz}$ noise from the signal.

The next filter that needs to be designed is the high pass and low pass filters. With these two filters in the circuit, it creates a band-pass filter (the band will be the frequency range of the bioelectric signal). As mentioned before, it is critical that this range of frequencies 
corresponds to the range of frequencies of the measured bioelectric signal. When designing the overall circuit, commonly the high pass filter is placed before the low pass filter.

High pass filter design is quite straightforward. Since a high pass filter will pass any frequency above the cut off frequency, the filter theoretically has an infinite frequency response. As such, if one was to design an active high pass filter, op amp utilized in the design may limit this response, as the op amp has a maximum frequency output. Therefore from a theoretical view, a passive filter will have the best response. In all practicality, this is not the case, but high pass active filters are still important to use, as they still can be effective. Depending on the bioelectric signal being measured, a simple RC passive filter can be sufficient. An example of a passive high pass filter is displayed in Figure 4.

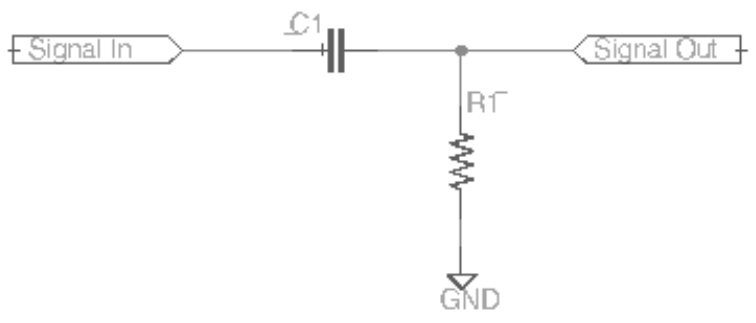

Fig. 4. Passive high pass filter

There are several possible common designs for the low pass filter. These include both active and passive filters. For these filters, there are several common types, including: Butterworth and Chebyshev. Each filter type has several configurations, with both active and passive designs. More commonly, for the low pass filter, an active configuration is utilized. As well, the Butterworth filter is typically used as it has an advantageous flat frequency response. There is also a choice of the filter order and configuration of the Butterworth Filter. Figure 5 shows a first order Butterworth low pass filter.

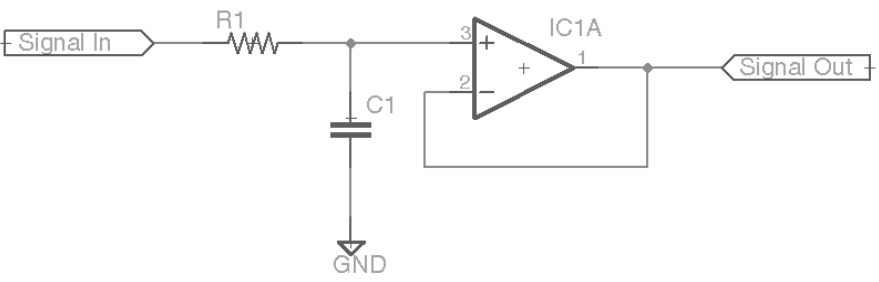

Fig. 5. First Order Butterworth Low Pass Filter

Typically, a filter with only a few orders will be utilized due to speed, cost, and space. A first order low pass Butterworth filter is perfectly acceptable choice for most applications. Another designer choice is the configuration of the filter. For active Butterworth filter, the Sallen-Key topology is an excellent choice. It allows for multiple orders, using only two op amps and several passive components. The design for the third order Butterworth filters is quite complex, and it involves calculating all values for the resistors and capacitors. There are several software packages that aide in the creation of these complex active filters. 


\subsubsection{Cascading filters and amplifiers}

Now that both amplifiers and filters have been discussed and designed, the next step is the layout of the circuit in the most logical order. When designing circuitry that filters and amplifies a signal, there is a general rule of thumb to alternate stages of amplification and filtering. This is critical since one will introduce less noise into the circuitry as well as amplify less of the noise. This is why the design requires the cascading of op amps and filters to alternate. Now, depending on the amount of gain that is required for the bioelectric signal, the following order can be used:

- Instrumentation Amplifier

- High Pass Filter

- Gain Stage

- Low Pass Filter

- Gain Stage

- Notch Filter

- Level Shift

Naturally, the instrumentation amplifier will also have some gain. Most instrumentation opamps have various levels of designable gain. To perform this type amplification, the de facto standard is to utilize a set of cascaded operational amplifiers. The need for cascaded stages will be explained later. When amplifiers are cascaded, one simply multiplies each of the gains together to determine the overall gain. Before designing the circuit, one needs to determine how much gain is actually necessary. The amount of gain will depend on what biometric signal is being measured, the ADC range, and other factors with that will vary from system to system.

\subsection{Power}

The voltage supply to the circuit components throughout the entire system is typically group together with the analog electronics design. Typically, there is a range of voltages are required through the system. For example, a microcontroller may require $5 \mathrm{~V}$, a wireless transceiver may require $3.3 \mathrm{~V}$, and the op-amps may require $+/-10 \mathrm{~V}$. It is critical to design a system to effectively convert the input voltage to these different voltage values. It is also important to determine the total power that is required by the loads. There is a lot of DC DC converters on the market, all of which have unique output power limits. In some cases it is perfectly acceptable to use voltage regulators instead of individual DC - DC converters.

\subsection{Safety issues}

With any electrical device that is being interfaced with a human, safety is a critical part of the hardware design. Not only do you have to be concerned about the damage a human can do to the device (i.e. ESD) but also the harm the device can make to human. In the case of a wireless transmitting biomedical device, over voltage protection is not as important to the device as opposed to when the device is connected directly to the computer. This is because the wireless transmitter acts as an isolated buffer between the patient and the monitoring computer or device. This way, the highest voltage in the device will be the source, typically a low voltage battery. Even at the low voltages of a battery, some protection is necessary for the device. Typically, this is performed using diodes that are designed into the circuit to only conduct when there is an over-voltage event. Naturally, these diodes are placed near the inputs, such that it is the first/last components before passing to the patient. This way, 
when the voltage is above the forward break down voltage ( $0.7 \mathrm{~V}$ for a silicon diode) the diode will then conduct. Since bioelectric signals have such small amplitude, the diodes do not disturb their signal.

\section{Digital hardware design}

\subsection{Microcontroller and digital hardware design}

The digital hardware system has three major parts: Microcontroller Unit (MCU), In System Programmer (ISP), and a Wireless Module (WM). The MCU includes a built in analog to digital converter (ADC). The ISP provides that capability to update the code on the MCU that is already a part of the board. Finally, the wireless module is involved in wireless data transmission. This communication is typically performed using Bluetooth. An illustration of this system is shown in Figure 6.

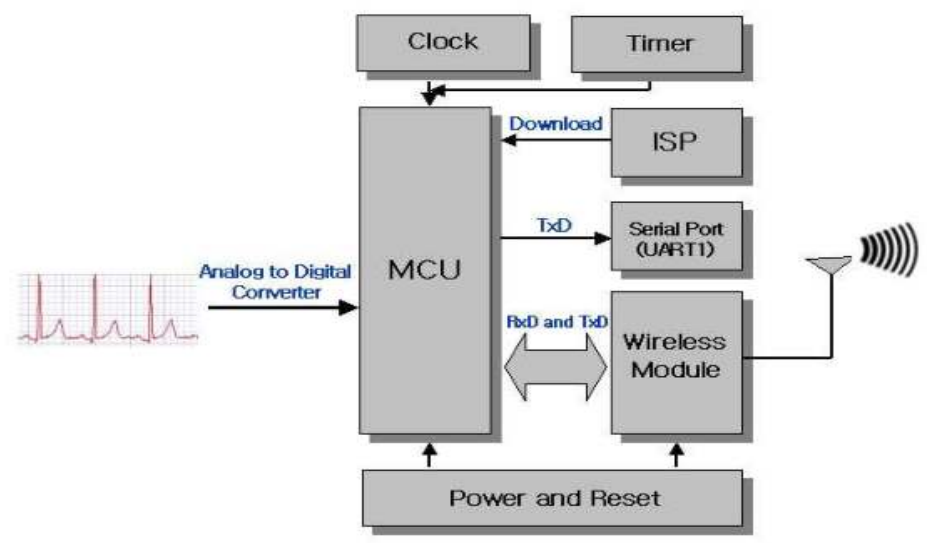

Fig. 6. Digital hardware block diagram

The MCU utilizes an 8-bit Reduced Instruction Set Computer (RISC), which has the advantages of simple commands, fast working speed, and low power consumption (2.7 5.5V). For example, the Atmel ATmega 128L has 16 Million Instructions per Second (MIPS) of performance. In addition, the ATmega MCU has $128 \mathrm{~KB}$ of In-System Programmable Flash with Read-While Writing capability. This is a type of flash memory that is 4 to 12 times faster than a general MCU. The ADC is responsible for converting continuous analog signals to digital signals. The ATmega MCU ADC has 8 channels and 10 bit resolution. This MCU also supports 16 different voltage input combinations and fast conversion time of 13 260us. Naturally, all MCUs are different, and these specifications will vary from MCU to MCU.

The ISP is the physical interface for programming the code on flash memory and EEPROM on the microcontroller. This hardware interface uses three signal lines: Master-Out-Slave-In (MOSI), Master-In-Slave-Out (MISO), and Clock (CLK). Once the reset pin on the microcontroller is set low, the code will updated via the ISP.

There are two possibilities to transmit the data from the microcontroller to a display device. One way to do this is via serial communication through the MAX232 IC. This IC will convert a TTL or CMOS signal into serial communication voltage level. This transmitting option 
requires a serial port and cable to transmit the data. In this sense, the communication is wired. Another option to transmit the data is via a wireless connection. This wireless communication is typically performed using a Bluetooth connection. This connection is created using a Bluetooth wireless module. In later sections, Bluetooth will be explored further. Other forms of wireless communication can be used, as per the system's requirements.

\subsection{Wireless system characteristics}

Wireless system (Bluetooth) uses a $2.4 \mathrm{GHz}$ band for short distance and low power consumption communication. Bluetooth is used for its high reliability and low cost. It is supported by AT-Command and has a transfer rate of approximately $1 \mathrm{Mbps}$ to $3 \mathrm{Mbps}$. Another feature of Bluetooth is its ability to guarantee stable wireless communication, even under severe noisy environment, by use of Frequency Hopping Spread Spectrum (FHSS). Bluetooth utilizes a packet based protocol with a master slave configuration. This configuration allows a wireless system to connect and communication to up to 7 devices. The terminology of master and slave is very straight forward. One device has control over one or more devices. In this application, the wireless transmitter has control over the wireless receiver. During communication, the transmitter first selects and pools the slave. This is termed the "inquiry" stage of communication, as master is determining the devices that it can connect to. Once the master pools the slave, the slave responds to the communication. Then the communication enters the "paging" phase that allows for the devices to synchronize the clock and frequency between the master and the slave. Once the master and slave modules are paired, the master module provides information to slave module with the master module's address. Once this is complete, the communication between devices can begin. The specifications for Bluetooth 2.0 are listed in Table 1.

\begin{tabular}{|c|c|}
\hline PART & Specification \\
\hline Bluetooth Spec. & Bluetooth Specification 2.0 Support \\
\hline Communication distance & $10 \mathrm{M}$ \\
\hline Frequency Range & $2.4 \mathrm{GHz}$ ISM Band \\
\hline Sensitivity & $-83 \mathrm{dBm}$ (Typical) \\
\hline Input Power & $3.3 \mathrm{~V}$ \\
\hline Current Consumption & $48 \mathrm{~mA}(\mathrm{Max})$ \\
\hline Communication Speed & $1,200 \mathrm{bps} \sim 230.400 \mathrm{bps}$ \\
\hline Antenna & Chip Antenna \\
\hline Interface & UART(TTL Level) \\
\hline
\end{tabular}

Table 1. Bluetooth 2.0 specifications

To perform the Bluetooth communication, a Bluetooth Module is utilized. This transceiver allows for communication between microcontroller and display device. For example, a WINiZEN Bluetooth RS232 wireless module is used for the Bluetooth communication. The data transmission and receiving power consumption of this Bluetooth module is 18 to $30 \mathrm{~mA}$ for transmission and $21 \sim 33 \mathrm{~mA}$ for reception. Data transmission/reception is achieved for up to 10 meter distances. The WINiZEN Bluetooth module is operated at low power (3.3 V), and has the dimensions of 18x20x12 (mm). This size can be visualized in Figure 7. 


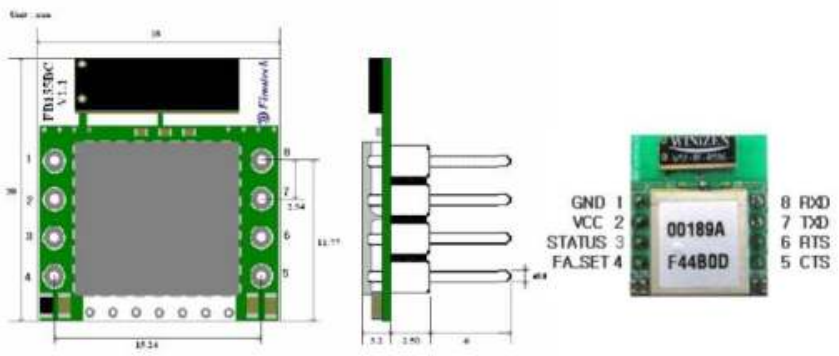

Fig. 7. Bluetooth Module Dimensions and Pin Assignments

The advantage of this, and any other small Bluetooth module, is the internal chip antenna that is used for short distance wireless communication. This is important since the transmitter or receiver device does not require an external antenna.

\subsection{Firmware and data communication}

The development of the firmware for the microcontroller is based upon the microcontroller that is being utilized in the design. As well, the code can be written in a verity of languages, typically Assembly and $C$, which again is dependent on the microcontroller selected. To continue the example microcontroller that was used in previous sections, Atmel ATmega microcontroller code was created using AVR Stdio4.0 and AVR ISP. The code was written in the C-language. AVR Stdio4.0 (Atmel Co., Ltd) which is a professional Integrated Development Environment (IDE) is used for writing, simulation, emulation and debugging. As a compiler, it also changes the firmware code from C-language to Hex code. An example firmware flow-charts for a biomedical signal transceiver is illustrated in Figure 8.

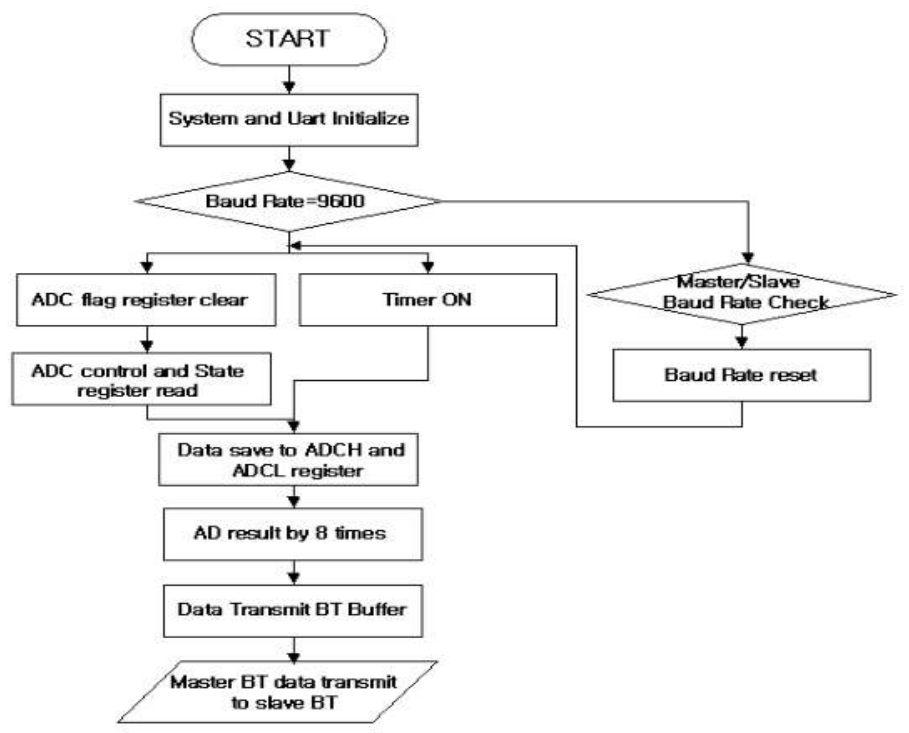

Fig. 8. Firmware Block Diagram 
The digital hardware works as follows. Once data from analog hardware circuitry has been amplified, filter, and shifted, the signal is sent to the ADC on the microcontroller. After the signal has been sampled, the microcontroller then sends the digital signal to the Bluetooth module via UART communication protocol. Then, the biomedical signal is transmitted wirelessly through wireless Bluetooth module, utilizing the master-slave configuration.

\section{ECG signal processing: A practical approach}

As an example of biomedical signal processing in a transceiver device, in this section, ECG signal analysis for real-time heart monitoring will be discussed. Following the discussion on previous sections, this section particularly describes where real-time ECG monitoring using hand-held devices, such as, smart phones or custom-designed health monitoring system. ECG analysis is one of the very first areas of physiological measurement where computer processing was introduced successfully. ECG has been widely used as the diagnostic measurement for heart monitoring. Typically cardiologists used to interpret the ECG tracing for identifying abnormalities related to function of heart. With the advancement in biomedical signal processing, it is now reality that smart monitoring system can mimic the rules cardiologists applies in order to monitor heart health in real time. Signal Processing and machine learning techniques plays an important role in this purpose. Hence, this type of smart devices would be useful in monitoring patient's health in a more efficient way (Laguna et al., 2005).

In the previous sections, we have described a low-cost real-time biomedical signal transceiver/monitoring system which could also use ECG as one of the physiological parameter to monitor health.

In the following sub-sections, we will discuss the underlying ECG signal processing techniques used for identifying heart abnormalities. Typical steps are preprocessing of ECG, wave amplitude and duration detection, heart rate calculation, ECG feature extraction algorithms, and finally identifying the abnormalities. A typical block diagram is illustrated in following Figure 9 (Neuman, 2010).

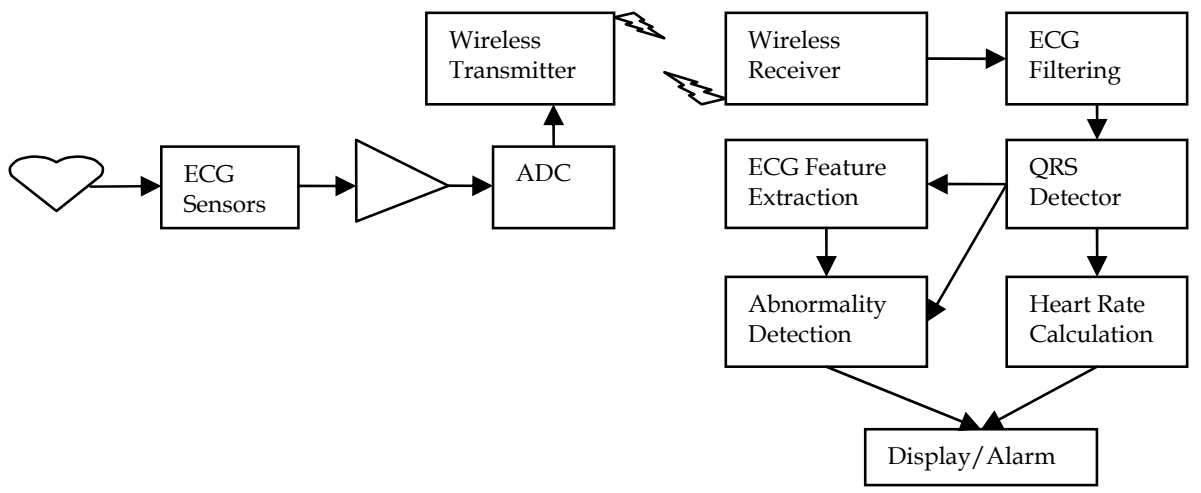

Fig. 9. Block diagram of biomedical signal transceiver for real-time acquisition, processing and heart condition monitoring system. The right portion of the system can be implemented in smart phone or PDA for smart health monitoring. 


\subsection{ECG digital filtering}

ECG signal is usually corrupted with different types of noise. These are baseline wander introduced during data acquisition, power line interference and muscle noise or artifacts as discussed in the pervious sections. Prior to applying different signal processing techniques for extraction different ECG features which are of clinical interests, data needs to be filtered in order to reduce noise and artifacts. Some of these filtering techniques are discussed below with a practical approach where the methods can be easily implementable (Laguna et al., 2005, Clifford, 2006).

\subsubsection{Power line interference}

Appropriate shielding and safety consideration could be employed to reduce this particular type of noise in addition to analog filtering discussed in previous sections. After receiving signals at the receiver sides, it is preferred to get rid of this type of noise in the preprocessing step (Laguna et al.,2005). Typically, band-stop (notch) filtering with cutoff, $\mathrm{F}_{\mathrm{C}}=50 / 60 \mathrm{~Hz}$ would suppress such noise. Figure 10 illustrates the magnitude and phase response of a digital second order Infinite Impulse Response (IIR) notch filter with cutoff frequency of $60 \mathrm{~Hz}$.
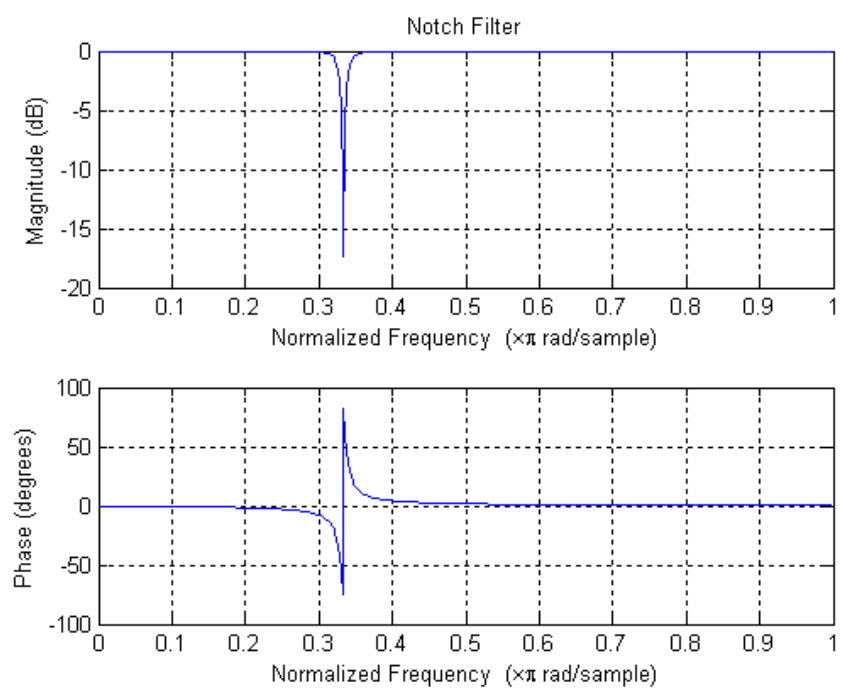

Fig. 10. Magnitude and phase response of an Infinite Impulse Response (IIR) notch filter to remove $60 \mathrm{~Hz}$ power line noise.

\subsubsection{Baseline wanders correction}

Due to the error in data collection process, baseline as well as dc offsets could be introduced. Removal of baseline wander is required prior to further processing. There are several techniques that have been widely used such as filtering and polynomial curve fitting method (Laguna et al. 2005, Clifford, 2006). Since all data processing starts with visual inspection, as for offline ECG analysis, we should inspect the data for baseline wander and dc bias. DC bias can easily removed by subtracting the mean from the original signal. As for 
the baseline wander corrections, filtering method is most widely used. Here, we will describe the linear filtering method to remove the baseline wander in ECG signals.

The frequency of the baseline wander is usually below $0.5 \mathrm{~Hz}$ (Laguna et al. 2005). This information particularly helps in design a high-pass filter in order to get rid of baseline wander. The design of a linear time-invariant high pass filter requires several considerations, most importantly, the choice of cut-off frequency and filter order. The ECG characteristic wave frequencies are higher than baseline wander. Therefore, a carefully designed high pass filter with cut-off frequency, $F_{\varepsilon}=0.5 \mathrm{~Hz}$ can effective remove the baseline. Baseline wander correction using a linear digital filter is shown in Figure 11. Instead of a high-pass filter we used band-pass filtering. We applied a sixth order butterworth filter with cutoff $0.7-40 \mathrm{~Hz}$. To avoid distortion, zero phase digital filtering was performed by processing the data in both forward and reverse direction. This can be done using MATLAB command filtfilt.

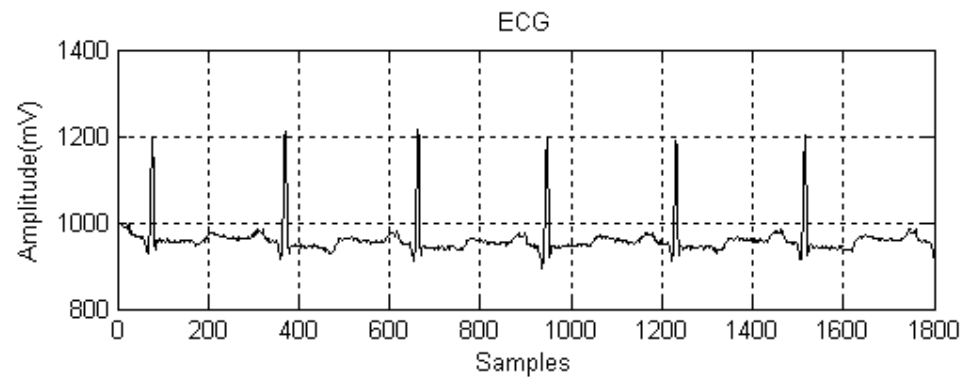

Bandpassed filtered ECG

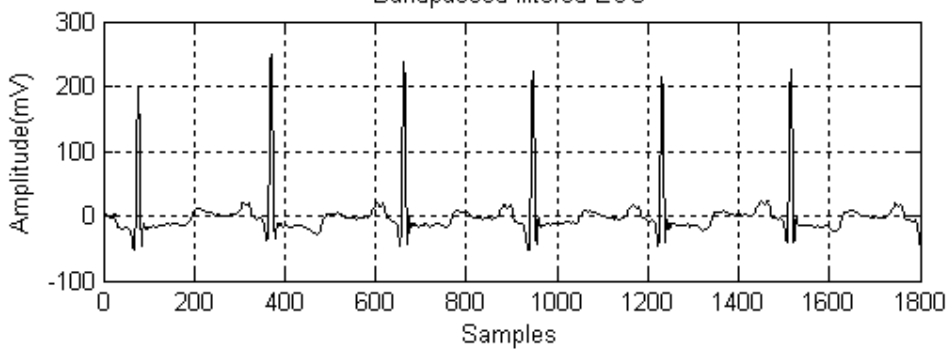

Fig. 11. ECG signal after band-pass filtering which effectively corrects baseline wander and reduces low frequency noise and high frequency artifacts. Prior to applying bandpass filter the DC components were removed from ECGs.

\subsection{QRS detection}

Detection of QRS complex is particularly most important in ECG signal processing. The information obtained from QRS detection, temporal information of each beat and QRS morphology information can be used for the improvement of performance for the other algorithms. In literature, many methods have been developed for detection of QRS complex. Most of these are based on filtering, nonlinear transformation and decision rules as well as using template matching method. In recent approaches, wavelet transform and empirical mode decomposition based algorithms have been developed. We will discuss a widely used 
and robust real-time QRS detection algorithm popularly known as Pam-Tompkins algorithm (Pan \& Tompkins, 1985).

The Pan-Tompkins algorithm consists of several steps. This is a single channel detection method in order to achieve better performance. In order to attenuate noise, the signal is first passed through a digital band-pass filter composed of cascaded high-pass and low-pass filters (Pan \& Tompkins, 1985). The band-pass filtering corrects the baseline wander, reduces muscle noise, $60 \mathrm{~Hz}$ power line interference, T-wave interference (Pan \& Tompkins, 1985). The cutoff frequency of this band-pass filter is an important design parameter. Another reason of this filtering is to maximize the energy of QRS complex. Pan and Tompkins suggested choosing $5-15 \mathrm{~Hz}$ as desirable pass band. The next steps involve nonlinear transformation in order to highlight the QRS complex from baseline. These steps are differentiation, squaring followed by moving window integration. Derivative stage provides the slope information of QRS complex. Squaring operation intensifies the slope of the frequency response curve. It also helps to restrict the false positives caused by unusually high amplitude $\mathrm{T}$ waves. The signal produced by the moving window integration operation provides the slope and width of the QRS complex. The choice of window sample size is an important parameter. Generally, it should be chosen such a way that the window size is same as the QRS width. The window width can be determined empirically making sure that QRS complex and $\mathrm{T}$ waves do not merge together. For practical application, we choose window of 30 samples (150 ms, sampling frequency was 360 samples/s). Finally, an adaptive threshold will be applied to identify the location of QRS complexes (Pan \& Tompkins, 1985).
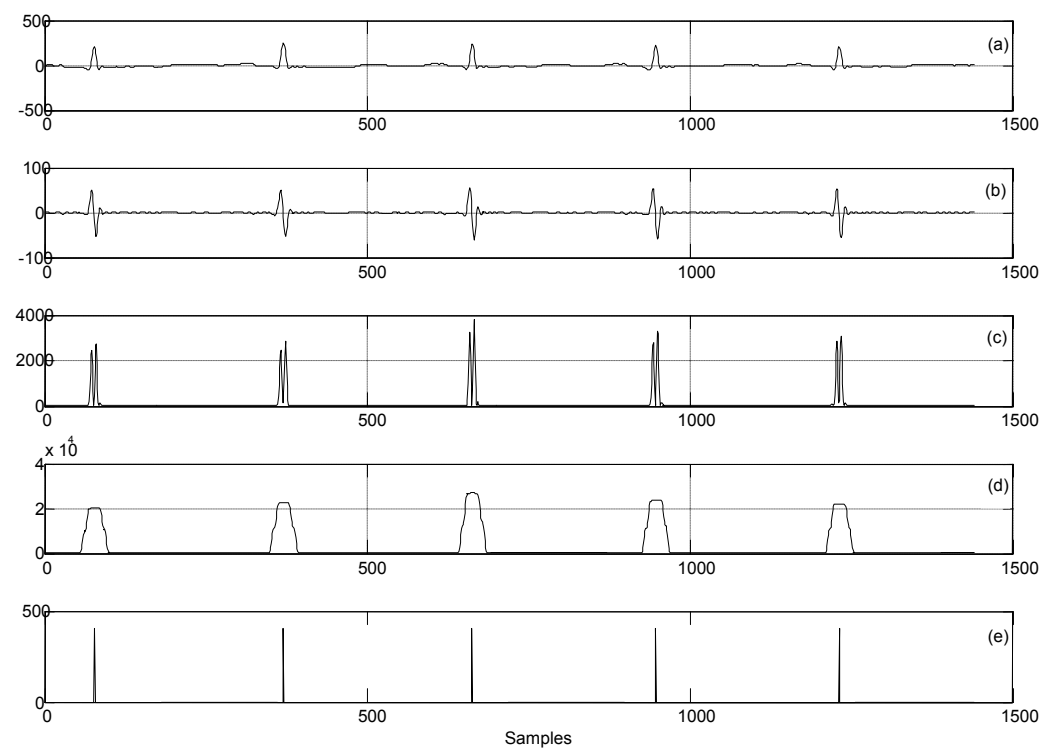

Fig. 12. Typical steps of Pan-Tompkins algorithm for detecting QRS complex: (a) band-pass filtered ECG signals; (b) after differentiation; (c) after performing squaring operation; (d) moving window integration; and (d) R peak detection. 
The fiducial points of the QRS complex can be detected from the integration waveform. The time duration of the rising edge is equal to the QRS width. The maximum slope is the location of $\mathrm{R}$ wave peak. $\mathrm{Q}$ and $\mathrm{S}$ points are the start and end points of the rising slope respectively (Pan \& Tompkins, 1985). The R peak detection is illustrated in Figure 12.

\subsection{Heart rate computation and display}

Heart rate is a vital sign to determine the patient's condition or well being. It can be easily computed from ECG (Neuman, 2010). Heart rate can be computed using various methods, for example, over one minute time period simply by counting the number of heart beats would give us the heart rate in beats per minute. However, if the QRS or R wave peak detector misses some beats that would adversely affect the heart rate and give wrong counts. The heart rate monitoring tool should avoid such wrong results. Therefore, it is more appropriate to measure the time duration between two successive $\mathrm{R}$ peaks known as $\mathrm{R}-\mathrm{R}$ intervals and computing instantaneous heart rate directly from $\mathrm{R}-\mathrm{R}$ interval. In clinical settings, heart rate is measured in beats per minute (bpm). So the formula for determining heart rate from RR interval is as following (Neuman, 2010).

$$
\text { Heart rate }(\mathrm{bpm})=\frac{60,000}{\text { RRinterval }(\mathrm{ms})}
$$

Once the program computes the heart rate it can be presented on the display of the monitoring device. We have discussed a low cost biomedical signal transceiver, where ECG signal is one parameter for health monitoring. Using this wireless transceiver ECG signal can be transmitted to a hand-held device, such as, smart phone or PDAs. The smart phone will have applications capable of computing instantaneous heart rate or average heart rate of a pre-determined period of time and shown as a digital output in the display of the smart phone. The application would also be able to identify primary abnormalities such as premature atrial contraction (APC or PAC) or premature ventricular contraction (PVC) and present an auditory or visual alarm to the patient.

\section{4 $P$ and $T$ wave detection}

Once QRS locations are identified, two other fudicial marks in ECG, P and T wave peak can be detected using the $\mathrm{R}$ peak location information. For detecting $\mathrm{T}$ wave onset, peak and offset, we should define a search window in forward direction. The size of the search window can be function of heart rate calculated. For detecting P wave, a backward search is required. Search window size can be determined similarly (Laguna et al., 1994).

Laguna et al. (1994) proposed an automatic method of wave boundaries detection in multilead ECG. The algorithm includes a QRS detector for multilead ECG, P and T wave detection. Since, a robust method of QRS detector for single channel has already been discussed in previous sub-sections here we focus mainly on $\mathrm{P}$ and $\mathrm{T}$ wave detection. Moreover, this method is also based on the QRS detection method of Pan and Tompkins. In preprocessing steps, a second order linear bandpass filter (cutoff frequency of 0.8-18 Hz, $3 \mathrm{~dB}$ ) is applied to the signal to remove baseline wander and attenuate high frequency noise. This signal is called ECGPB. Then a low-pass differentiator (Pan \& Tompkins, 1985) is applied to obtain the information about changes in slope. This differentiated signal is called 
ECGDER. Then moving-window integration will be performed. In this case, Laguna et al. had chosen the window size of $95 \mathrm{~ms}$. Since $\mathrm{P}$ and $\mathrm{T}$ waves usually have lower frequency components than the QRS complex, a digital low-pass filter (cutoff frequency of $12 \mathrm{~Hz},-3 \mathrm{~dB}$ ) is applied to the ECGDER signal. This filtering reduces the remaining noise and highlights the $\mathrm{P}$ and $\mathrm{T}$ wave components and the signal is called (DERFI) (Laguna et al. 1994). Now a search window of $150 \mathrm{~ms}$ is defined which starts $225 \mathrm{~ms}$ before the R wave position. In this search window, the algorithm searches for maximum and minimum values. The $\mathrm{P}$ wave peak is assumed to be occurred at the zero-crossing of the maximum and minimum values (Laguna et al., 1994).

Similarly for $\mathrm{T}$ wave detection, a search window is defined which is a function of heart rate already calculated. In this search window, the algorithm searches for maximum and the minimum values. However, the $\mathrm{T}$ wave peak is assumed to be occurred at the zero-crossing of the maximum and minimum values. The $\mathrm{T}$ wave morphology varies hugely. The algorithm can also identify the type of $\mathrm{T}$ wave (for example, regular or inverted) by examining the relative position and values of the maximum and minimum values in the search window (Laguna et al., 1994).

\section{References}

B. Chi, J. Yao, S. Han, X. Xie, G. Li, Z. Wang, "Low-Power Transceiver Analog Front-End Circuits for Bidirectional High Data Rate Wireless Telemetry in Medical Endoscopy Applications," Biomedical Engineering, IEEE Transactions on , vol.54, no.7, pp.1291-1299, July 2007 doi: 10.1109/TBME.2006.889768

G. D. Clifford. Advanced Methods and Tools for ECG Data analysis. Cambridge, Massachusetts, 2006.

J. H. Schulman, P. Mobley, J. Wolfe, H. Stover, A. Krag, “A 1000+ Channel Bionic Communication System," Proceedings of the 28th IEEE EMBS Annual International Conference, New York City, USA, Aug. 30 - Sept. 3, 2006.

J. M. R. Delgado, "Electrodes for Extracellular Recording and Stimulation," in Physical Techniques in Biological Research, W.L. Nastuk, Ed., New York: Academic Press, 1964.

J. Pan and W. J. Tompkins, A Real-Time QRS Detection Algorithm" IEEE Transactions on Biomedical Engineering vol. 32(3), pp. 230-236, 1985.

J. Molina, Design a $60 \mathrm{~Hz}$ Notch Filter with the UAF42, Burr-Brown Application Bulletin,11/30/10, <http://focus.ti.com/lit/an/sbfa012/sbfa012.pdf>

L. Sornmo, P. Laguna. Bioelectric Signal Processing in Cardiac and Neurological Applications. Elsevier Academic Press, 2005.

M. R. Neuman, “Vital Signs: Heart Rate,” IEEE Pulse, November/December 2010, vol. 1(3), pp.51-55. 2010.

P. Laguna, R. Jane, and P. Caminal, "Automatic detection of wave boundaries in multilead ECG signals: validation with the CSE database," Computers and biomedical research, vol. 27(1), pp. 45, 1994. 
R. R. Harrison, P. T. Watkins, R. J. Kier, R. O. Lovejoy, D. J. Black, B. Greger, F. Solzbacher, "A Low-Power Integrated Circuit for a Wireless 100-Electrode Neural Recording System," IEEE Journal of Solid State Circuits, Vol. 42, No. 1, Jan. 2007.

A. S. Sedra and A. C. Smith. Microelectronic Circuits. New York: Oxford UP, 2010. 


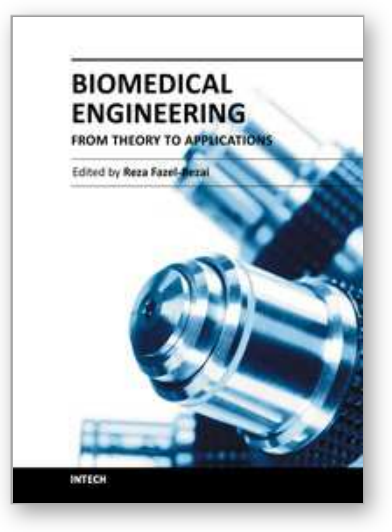

\author{
Biomedical Engineering - From Theory to Applications \\ Edited by Prof. Reza Fazel
}

ISBN 978-953-307-637-9

Hard cover, 486 pages

Publisher InTech

Published online 29, August, 2011

Published in print edition August, 2011

In all different areas in biomedical engineering, the ultimate objectives in research and education are to improve the quality life, reduce the impact of disease on the everyday life of individuals, and provide an appropriate infrastructure to promote and enhance the interaction of biomedical engineering researchers. This book is prepared in two volumes to introduce a recent advances in different areas of biomedical engineering such as biomaterials, cellular engineering, biomedical devices, nanotechnology, and biomechanics. It is hoped that both of the volumes will bring more awareness about the biomedical engineering field and help in completing or establishing new research areas in biomedical engineering.

\title{
How to reference
}

In order to correctly reference this scholarly work, feel free to copy and paste the following:

Reza Fazel-Rezai, Noah Root, Ahmed Rabbi, DuckHee Lee and Waqas Ahmad (2011). Biomedical Signal Transceivers, Biomedical Engineering - From Theory to Applications, Prof. Reza Fazel (Ed.), ISBN: 978-953307-637-9, InTech, Available from: http://www.intechopen.com/books/biomedical-engineering-from-theory-toapplications/biomedical-signal-transceivers

\section{INTECH}

open science | open minds

\section{InTech Europe}

University Campus STeP Ri Slavka Krautzeka 83/A 51000 Rijeka, Croatia Phone: +385 (51) 770447

Fax: +385 (51) 686166 www.intechopen.com

\section{InTech China}

Unit 405, Office Block, Hotel Equatorial Shanghai No.65, Yan An Road (West), Shanghai, 200040, China 中国上海市延安西路65号上海国际贵都大饭店办公楼 405 单元 Phone: +86-21-62489820

Fax: +86-21-62489821 
(C) 2011 The Author(s). Licensee IntechOpen. This chapter is distributed under the terms of the Creative Commons Attribution-NonCommercialShareAlike-3.0 License, which permits use, distribution and reproduction for non-commercial purposes, provided the original is properly cited and derivative works building on this content are distributed under the same license. 